\title{
COMPLETE LOCALIZATION OF DOMAINS WITH NONCOMPACT AUTOMORPHISM GROUPS
}

\author{
KANG-TAE KIM
}

\begin{abstract}
We prove a characterization of the domains in $\mathbf{C}^{n}$ with an automorphism orbit accumulating at a boundary point at which the boundary is real analytic and convex up to a biholomorphic change of local coordinates. This result generalizes the well-known Wong-Rosay theorem on strongly pseudoconvex domains to the case of locally convex domains with real analytic boundaries.
\end{abstract}

\section{INTRODUCTION}

For a given pseudoconvex domain $\Omega$ in $\mathbf{C}^{n}$ we call $p \in \partial \Omega$ a local peak point if there is a continuous function $f: U \rightarrow \mathbf{C}$ defined on an open neighborhood of $p$ in $\mathbf{C}^{n}$ satisfying:

(1) $f$ is complex analytic on $U \cap \Omega$;

(2) $f(p)=1$, and $|f(q)|<1$ for any $q \in \operatorname{closure}(\Omega) \backslash\{p\}$.

The function $f$ defined above is called a local peaking function of $\Omega$ at $p$. We also denote by $\operatorname{Aut}(\Omega)$ the group of all biholomorphic mappings from $\Omega$ onto itself. Then as J.-P. Rosay proved in [7], we have the following phenomenon:

If there are a sequence $\left\{g_{j}\right\} \subset \operatorname{Aut}(\Omega)$, a local peak point $p \in \partial \Omega$ and a point $p_{0} \in \Omega$ such that $g_{j}\left(p_{0}\right) \rightarrow p$ as $j \rightarrow \infty$ in $\mathbf{C}^{n}$, then for any compact subset $K \subset \subset \Omega$ and for any $\varepsilon>0$, there exists $j_{0}>0$ such that

$$
g_{j}(K) \subset \subset B_{\varepsilon}(p) \cap \Omega \quad \text { for any } j \geq j_{0},
$$

where $B_{\varepsilon}(p)$ denotes the open ball of radius $\varepsilon$ centered at $p$ in $\mathbf{C}^{n}$.

Therefore, in principle, the relatively small part, $B_{\varepsilon}(p) \cap \Omega$ of $\Omega$, should be enough to understand the whole domain $\Omega$. This is, of course, only a highly philosophical statement. However, in the case of strongly pseudoconvex domains, as in [7,8], this idea has a precise form, the following well-known

Wong-Rosay theorem. Any bounded domain $\Omega \subset \subset \mathbf{C}^{n}$ with a $C^{2}$ strongly pseudoconvex boundary point $p \in \partial \Omega$, admitting a sequence $\left\{g_{j}\right\} \subset$ Aut $\Omega$ such that

Received by the editors March 15, 1989.

1980 Mathematics Subject Classification (1985 Revision). Primary 32F15, Secondary 32A40. 
$g_{j}\left(p_{0}\right) \rightarrow p$ as $j \rightarrow \infty$ for some $p_{0} \in \Omega$, is biholomorphic to the open unit ball in $\mathbf{C}^{n}$.

This beautiful theorem has played an important role in the geometric theory of strongly pseudoconvex domains in $\mathbf{C}^{n}$, as one finds from [3]. So it is natural to attempt to prove an analogue of this theorem in the case of weakly pseudoconvex domains. However, the techniques developed in [7,8] cannot be directly applied to the weakly pseudoconvex domains. R. Greene and S. Krantz [4] found out how to use the $C / K$ invariant, which was the major tool in [7], [8], for the characterization of the smooth Thullen domains defined by

$$
\left|z_{1}\right|^{2}+\left|z_{2}\right|^{2 m_{2}}+\cdots+\left|z_{n}\right|^{2 m_{n}}<1
$$

in $\mathbf{C}^{n}$, which possess noncompact automorphism groups but are not biholomorphic to the ball unless all $m_{j}$ are equal to 1 . This result is not a special case of the Wong-Rosay theorem, because these domains are weakly pseudoconvex. However, Greene and Krantz's proof is very complicated, since they needed to control the shape of the orbit of the sequences of automorphisms near the boundary points.

The goal of this paper is to extend the result of [4] just quoted to include a broader collection of domains. The basic method is a normal family argument which is a modification of the theorem in [2]:

The sequence of holomorphic mappings $\sigma_{j}(z): \Omega \rightarrow \mathbf{C}^{n}$ defined by

$$
\sigma_{j}(z):=\left[\partial g_{j}\left(p_{0}\right)\right]^{-1}\left(g_{j}(z)-p\right)
$$

is shown to be a normal family with all the subsequential limits one-to-one and onto, provided that the domain $\Omega$ and $\left\{g_{j}\right\} \subset$ Aut $\Omega$ satisfy the condition called Condition (L) at $p$, which is defined in $\S 2$ of this paper. Moreover, by choosing a subsequence if necessary, we obtain a local set convergence of the domains $\sigma_{j}(\Omega)$ in $\mathbf{C}^{n}$.

It turns out that this result is useful because we do not have to worry about how to control the shape of the orbit of the sequence at the boundary point. Meanwhile, we would like to mention that this method is closely related to the method introduced in [6]. In the present paper, analyzing the local Hausdorff limit of the sequence $\sigma_{j}(\Omega)$, we have shown that the limit domain is entirely determined by the shape of $\Omega$ near $p \in \Omega$, up to biholomorphic mappings, provided that the domain $\Omega$ satisfies the Condition (L). For the precise statements, see the main theorems in $\S 2$.

Note that there are many weakly pseudoconvex domains satisfying Condition (L). Thus our main result here is indeed more general than the result of the Wong-Rosay [7, 8]) and the result of Greene-Krantz ([4]). Of course, our statement, as it stands, is not a direct generalization of Wong-Rosay theorem. However, the Wong-Rosay theorem can be proved by our technique with suitable small modifications. We would like to express our deepest gratitude to 
R. E. Greene for valuable suggestions and encouragement. This work is part of the author's Ph.D. dissertation at the University of California at Los Angeles.

\section{DEFINITIONS AND STATEMENTS}

Definition. Let $\Omega$ be a bounded pseudoconvex domain in $\mathbf{C}^{n}$ with boundary $\partial \Omega$. A boundary point $p \in \partial \Omega$ is said to be convexifiable, if there exist an open neighborhood $U$ of $p$ in $\mathbf{C}^{n}$ and a one-to-one holomorphic mapping $f: U \rightarrow \mathbf{C}^{n}$ with $f(U \cap \Omega)$ convex.

We recall the definition of finite type from D'Angelo [1]:

Definition. Let $\Omega$ be a pseudoconvex domain in $\mathbf{C}^{n}$ with smooth boundary and with the defining function $\rho: \mathbf{C}^{n} \rightarrow \mathbf{R}$, i.e.,

$$
\begin{gathered}
\Omega=\left\{z \in \mathbf{C}^{n} \mid \rho(z)<0\right\}, \\
\mathbf{C}^{n} \backslash \Omega=\left\{z \in \mathbf{C}^{n} \mid \rho(z)>0\right\}, \\
\operatorname{grad} \rho(q) \neq 0 \text { for any } q \in \partial \Omega .
\end{gathered}
$$

Let $X$ be a germ of a complex analytic variety of complex dimension 1, represented by the analytic function

$$
\psi=\left(\psi_{1}, \ldots, \psi_{n}\right): V \rightarrow \mathbf{C}^{n}
$$

defined on an open neighborhood $V$ of $0 \in \mathbf{C}$ with $\psi(0)=p, \quad \psi \not \equiv p$, where $p \in \partial \Omega$. Let $\nu(f)$ denote the order of vanishing of $f$ at 0 for any smooth function $f$ defined on an open neighborhood of 0 in $\mathbf{C}$ mapping into $\mathbf{C}^{n}$. Then we define

$$
\tau^{*}(\partial \Omega, p, X)=\frac{\nu(\rho \circ \psi)}{\nu(\psi)}
$$

which is, roughly speaking, the order of contact of $X$ with $\partial \Omega$ at $p$. Now we define the type of $\partial \Omega$ at $p$ by the quantity

$$
\tau(\partial \Omega, p)=\sup \tau^{*}(X)
$$

where the supremum is taken over all germs $X$ of the complex varieties. Moreover, we say that $\Omega$ is of finite type $l$, if $\tau(\partial \Omega, p)=l<\infty$. It is known that the type is always an even number if the boundary of the domain is real analytic (cf. [1]).

Definition. We say that a bounded pseudoconvex domain $\Omega \subset \subset \mathbf{C}^{n}$ satisfies Condition (L) at $p \in \partial \Omega$, if the following three conditions hold:

(1) $\partial \Omega$ is real analytic near $p$ and is of finite type $2 k$ at $p$.

(2) $p \in \partial \Omega$ is convexifiable.

(3) There exist a point $p_{0} \in \Omega$ and a sequence $\left\{g_{j}\right\} \subset$ Aut $\Omega$ such that $\lim _{j \rightarrow \infty} g_{j}\left(p_{0}\right)=p$ in $\mathbf{C}^{n}$.

Then we prove the following main result. 
Theorem 1. Let $\Omega_{1}$ and $\Omega_{2}$ be bounded pseudoconvex domains in $\mathbf{C}^{n}$ satisfying the Condition (L) at $p_{1} \in \partial \Omega_{1}$ and at $p_{2} \in \partial \Omega_{2}$, respectively. If there exist open neighborhoods $U_{1}$ of $p_{1}$ and $U_{2}$ of $p_{2}$ in $\mathbf{C}^{n}$ and a biholomorphic mapping $f: U_{1} \rightarrow U_{2}$, with $f\left(p_{1}\right)=p_{2}$ with $f\left(U_{1} \cap \partial \Omega_{1}\right)$ coinciding with $U_{2} \cap \partial \Omega_{2}$ up to and including the $2 k$ th order terms in the Taylor expansions at $p_{2}$ of defining functions where $2 k=\tau\left(\partial \Omega_{1}, p_{1}\right)=\tau\left(\partial \Omega_{2}, p_{2}\right)$, then $\Omega_{1}$ and $\Omega_{2}$ are biholomorphic to each other.

Remark. This theorem, in particular, characterizes the smooth Thullen domains

$$
\left\{\left.\left(z_{1}, \ldots, z_{n}\right) \in \mathbf{C}^{n}|| z_{1}\right|^{2}+\left|z_{2}\right|^{2 m_{2}}+\cdots+\left|z_{n}\right|^{2 m_{n}}<1\right\}
$$

by their automorphism groups and the local shape of the boundaries near $(1,0$, $\ldots, 0)$.

The proof of Theorem 1 above yields the following result also:

Theorem 2. Let $\Omega$ be a bounded pseudoconvex domain in $\mathbf{C}^{n}$ satisfying the Condition $(\mathrm{L})$ at some $p \in \partial \Omega$. Then $\Omega$ is biholomorphic to the domain represented by the inequality

$$
0>\operatorname{Re} z_{1}+P_{m_{2}}\left(z_{2}\right)+\cdots+P_{m_{n}}\left(z_{n}\right)+\sum_{i_{2}, \ldots, i_{n} \geq 0} Q_{i_{2} \cdots i_{n}}\left(z_{2}, \ldots, z_{n}\right)
$$

where:

(1) $P_{m_{k}}(k=2, \ldots, n)$ are real valued positive homogeneous polynomials of degree $m_{k}$

(2) $Q_{i_{2} \ldots i_{n}}$ are either identically 0 , or real homogeneous polynomials of degree $\sum_{l=2}^{n} i_{l}$, with fixed degree $i_{l}$ in variables $z_{l}, \bar{z}_{l}$ for each $l$; and

(3) $\left(i_{2}, \ldots, i_{n}\right)$ varies over the set of $(n-1)$-tuples of nonnegative integers, at least two of whose entries are nonzero, satisfying the relation

$$
\frac{i_{2}}{m_{2}}+\cdots+\frac{i_{n}}{m_{n}}=1
$$

Corollary. For any bounded (or just complete hyperbolic) pseudoconvex domain satisfying Condition (L), its automorphism group has the noncompact identity component with respect to the compact-open topology.

Corollary. Any bounded pseudoconvex domain in $\mathbf{C}^{n}$ with real analytic boundary which is convexifiable at every point and possesses a noncompact automorphism group is biholomorphic to one of the domains defined by the inequality ( $\dagger)$.

While preparing this manuscript, it became known to us that E. Bedford and $\mathrm{S}$. Pinchuk had proved recently a much stronger result in complex dimension two. Their theorem is that every bounded pseudoconvex domain in $\mathbf{C}^{2}$ with a real analytic boundary and with a noncompact automorphism group is biholomorphic to one of the smooth Thullen domains. However, we believe that our theorem is of separate interest even in complex dimension two. In light of the 
Bedford-Pinchuk theorem, it would be very attractive if one could classify the homogeneous polynomials appearing in $(\dagger)$.

\section{PROOF OF THE MAIN THEOREMS}

We start the proof with

Lemma A. Let $\Omega$ be a bounded pseudoconvex domain in $\mathbf{C}^{n}$ satisfying the Condition (L) at $p \in \partial \Omega$. Then there is a sequence $\left\{A_{j}\right\} \subset G L_{n}(\mathbf{C})$ such that

(1) $\left\|A_{j}^{-1}\right\| \rightarrow 0$ as $j \rightarrow \infty$; and

(2) $\lim _{j \rightarrow \infty} A_{j}(\Omega-p)=\hat{\Omega}$ exists and is biholomorphic to $\Omega$,

where the limit is taken in the sense of local Hausdorff distances in $\mathbf{C}^{n}$ and where $\Omega-p=\left\{z-p \in \mathbf{C}^{n} \mid z \in \Omega\right\}$.

Proof. This lemma is a modification of the theorem of S. Frankel [2] which implies that the sequence $\left\{\omega_{j}: \Omega \rightarrow \mathbf{C}^{n}\right\}$, where $\omega_{j}$ are defined to be $\omega_{j}(z):=$ $\left[d g_{j}\left(p_{0}\right)\right]^{-1}\left(g_{j}(z)-g_{j}\left(p_{0}\right)\right)$ forms a normal family. Moreover, any subsequential limit is biholomorphic. Hence, we begin with

Step 1. The proof of the statement above:

It is enough to estimate $\left\|d \omega_{j}\right\|_{\text {sup }}$ from above on each compact subset of $\Omega$. To make the proof as clear as possible we introduce the following notations:

$$
A_{j}(z):=\left[d g_{j}\left(p_{0}\right)\right]^{-1}\left(z-g_{j}\left(p_{0}\right)\right), \quad \varphi(z, \zeta):=\frac{z+\zeta}{2}
$$

for any $z, \zeta \in \mathbf{C}^{n}$.

Let $K_{0}$ be an arbitrary compact subset of $\Omega$. Then there is another compact subset $K$ of $\Omega$ which contains $K_{0}$ in its interior. Choose $r>0$ such that $B_{r}(p) \cap \Omega$ is convex after a holomorphic change of coordinates at $p$. We also assume, momentarily, that $B_{r}(p) \cap \Omega$ is actually convex. (After carrying out the proof, it should be clear that we may assume this without loss of generality.) By (1) and (2) in Condition (L), there is a local peaking function of $\Omega$ at $p$, and hence there exists $j_{0}>0$ such that $g_{j}(K) \subset B_{r}(p) \cap \Omega$ for any $j \geq j_{0}$ (cf. [7]). Thus we may assume that $g_{j}(K) \subset B_{r}(p) \cap \Omega$ for all $j$.

Now we consider the mapping $F_{j}: K \times K \rightarrow \Omega$ defined by the following diagram:

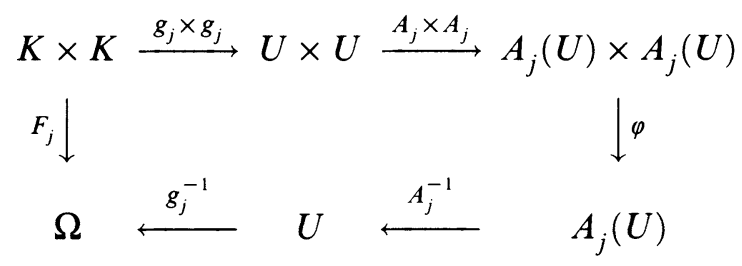

where $U:=B_{r}(p) \cap \Omega$. Namely, $F_{j}(z, \zeta)=\omega_{j}^{-1} \circ \varphi \circ\left(A_{j} \times A_{j}\right) \circ\left(g_{j} \times g_{j}\right)$. By Kobayashi [5, p. 74], $\left\{F_{j}\right\}$ is a normal family of holomorphic mappings, since $F_{j}(0,0)=0$ for all $j$. 
Now we look at

$$
\omega \circ F(z, \zeta)=\frac{\omega(z)+\omega(\zeta)}{2}
$$

(note that here we drop the subscripts momentarily) where $z=\left(z^{1}, \ldots, z^{n}\right.$ ) and $\zeta=\left(\zeta^{1}, \ldots, \zeta^{n}\right)$. A direct computation shows that

$$
\sum_{j} \sum_{m} \frac{\partial^{2} \omega^{l}}{\partial z^{m} \partial z^{j}}(\circ F) \frac{\partial F^{m}}{\partial z^{k}} \frac{\partial F^{j}}{\partial z^{i}}=-\sum_{j} \frac{\partial^{2} F^{j}}{\partial z^{i} \partial \zeta^{k}} \frac{\partial \omega^{l}}{\partial z^{j}}(\circ F) .
$$

Moreover,

$$
\sum_{m} \sum_{j} \frac{\partial^{2} \omega^{l}}{\partial z^{m} \partial z^{j}}(\circ F) \frac{\partial F^{m}}{\partial z^{k}} \frac{\partial F^{j}}{\partial z^{i}}+\sum_{j} \frac{\partial \omega^{l}}{\partial z^{j}}(\circ F) \frac{\partial^{2} F^{j}}{\partial z^{k} \partial z^{i}}=\frac{1}{2} \frac{\partial^{2} \omega^{l}}{\partial z^{k} \partial z^{i}} .
$$

Combining (A) and (B), we obtain, at $(z, z)$,

$$
\frac{1}{2} \frac{\partial^{2} \omega^{l}}{\partial z^{k} \partial z^{i}}(z)=\sum_{j}\left(\frac{\partial^{2} F^{j}}{\partial z^{k} \partial z^{i}}-\frac{\partial^{2} F^{j}}{\partial \zeta^{k} \partial z^{i}}\right)(z, z) \frac{\partial \omega^{l}}{\partial z^{j}}(z) .
$$

Since $\left\{F_{\lambda}\right\}_{\lambda}$ is a normal family as we pointed out, we have $\left\|D^{2} \omega_{j}\right\| \leq$ $C_{K_{0}}\left\|D \omega_{j}\right\|$ on $K_{0}$. Also we have $\omega_{j}\left(p_{0}\right)=0$ and $D \omega_{j}\left(p_{0}\right)=$ identity. Therefore, we get $\left\|D \omega_{j}\right\| \leq C_{K_{0}}^{\prime}$ for some constant $C_{K_{0}}^{\prime}$ depending only on $K_{0}$. So we have proven that $\omega_{j}$ is a normal family of holomorphic mappings.

Now, let $\omega=\lim _{k \rightarrow \infty} \omega_{k}$ be a subsequential limit of $\left\{\omega_{j}\right\}$. Then since the $\omega_{k}$ 's are biholomorphic and since $\omega_{j}\left(p_{0}\right)=0$, we can conclude that the determinant of the holomorphic Jacobian of $\omega$ is nowhere zero, by applying Hurwitz's theorem. It is also easy to see that $\omega$ has to be globally one-to-one since the convergence of $\left\{\omega_{j}\right\}$ is uniform on compact subsets.

Moreover, note that every eigenvalue of $d g_{j}(0)$ tends to 0 as $j$ approaches $\infty$, which can be seen as follows:

Suppose that there exists a vector $v \in \mathbf{C}^{n}$ such that

$$
\lim _{j \rightarrow \infty}\left\|d g_{j}(0) v\right\| \geq \varepsilon>0 .
$$

Choose a positive real number $\delta>0$, independent of $j$, such that $f^{\prime}(0)=v$ for some holomorphic mapping $f$ from the open disc in $\mathbf{C}$, with a certain radius $\delta$ and centered at 0 into $\Omega$, with $f(0)=0$. Then consider the sequence $\left\{g_{j} \circ f\right\}$ of holomorphic mappings. This is a normal family, since $\Omega$ is bounded. Let $\Phi$ be the limit of a subsequence of this normal family. Then the image of $\Phi$ is contained in the closure of $\Omega$. Since $\Phi(0)=p \in \partial \Omega$, the image of $\Phi$ will be entirely contained in the boundary, $\partial \Omega$, by the Maximum Principle. On the other hand, $\left\|\Phi^{\prime}(0)\right\| \geq \varepsilon>0$. Therefore, the boundary $\partial \Omega$ of $\Omega$ admits a nonconstant analytic set at $p$. But this is impossible because $p$ is a local peak point of $\Omega$. 
We have yet to prove that some subsequence of $\left[d g_{j}(0)\right]^{-1}\left(g_{j}(z)-p\right)$ converges uniformly on all compact subsets. To prove this we first need to show the following.

Step 2. We can choose a subsequence of $\left\{\omega_{j}\right\}$, which we again denote by $\left\{\omega_{j}\right\}$, such that $\left\{\omega_{j}(\Omega)\right\}$ converges to $\omega(\Omega)$ in the local Hausdorff distance (i.e., in the sense of local set convergence). Moreover, $\omega(\Omega)$ is convex.

This is easy to prove. From the definition of $\omega_{j}$ we have

$$
\omega_{j}(\Omega)=\left[d g_{j}\left(p_{0}\right)\right]^{-1}\left(\Omega-p_{j}\right)
$$

with $p_{j} \rightarrow p \in \Omega$ as $j \rightarrow \infty$, since $g_{j}(\Omega)=\Omega$. Now since all the eigenvalues of $\left[d g_{j}\left(p_{0}\right)\right]$ tend to 0 as $j \rightarrow \infty$, for any $R>0$, there exists $j_{R}>0$ such that

$$
B_{R}(0) \cap \omega_{j}(\Omega)=\omega_{j}\left(B_{r}(p) \cap \Omega\right) \cap B_{R}(0)
$$

for all $j>j_{R}$. Since $B_{r}(p) \cap \Omega$ is convex, $B_{R}(0) \cap \omega_{j}(\Omega)$ is convex for any $j>j_{R}$. Hence, by choosing subsequences, we have proven the conclusion of this step.

Thus it suffices to show

Step 3. The sequence $\sigma_{j}(z):=\left[d g_{j}\left(p_{0}\right)\right]^{-1}\left(g_{j}(z)-p\right)$ of holomorphic mappings from $\Omega$ to $\mathbf{C}^{n}$ is also a normal family. Moreover, any subsequential limit gives a holomorphic embedding of $\Omega$ into $\mathbf{C}^{n}$.

It is enough to show that $\left[d g_{j}\left(p_{0}\right)\right]^{-1}\left(p-p_{j}\right)$ is a bounded sequence in $\mathbf{C}^{n}$ where $p_{j}=g_{j}\left(p_{0}\right)$. For, once we get this, then the claim follows from Step 1 . Thus, suppose that $\lim _{j \rightarrow \infty}\left[d g_{j}\left(p_{0}\right)\right]^{-1}\left(t-p_{j}\right)=\infty$ for every boundary point $t$ of $\Omega$. Since $0 \in \omega_{j}(\Omega)$ for all $j$, the sequence of domains $\left\{\omega_{j}(\Omega)\right\}$ converges to the entire $\mathbf{C}^{n}$ in local Hausdorff distance. Therefore, we have $\omega(\Omega)=\mathbf{C}^{n}$ and hence $\Omega$ is biholomorphic to $C^{n}$ by Step 1. But this is impossible since $\Omega$ is hyperbolic. (Here, as usual, $\omega$ denotes $\lim _{j \rightarrow \infty} \omega_{j}$.) Therefore, there exists a $t_{0} \in \partial \Omega$ such that

$$
\left\|A_{j}\left(t_{0}\right)\right\| \leq C \text { for any } j
$$

with an absolute constant $C$ independent of $j$. Now we show that $t_{0}=p$. Note that we have $\left[d g_{j}\left(p_{0}\right)\right]^{-1} v \rightarrow \infty$ for any $v \neq 0$ in $\mathbf{C}^{n}$. Therefore, $t_{0}-p_{j} \rightarrow 0$ as $j \rightarrow \infty$. But we have $p_{j} \rightarrow p$ as $j \rightarrow \infty$. Hence, we conclude that $p=t_{0}$. So the proof of Lemma $\mathrm{A}$ is complete.

We try to apply Lemma $\mathrm{A}$ above, to $\Omega_{1}$ at $p_{1}$, to generate a "unique and canoriical" $\hat{\Omega}_{1}$, which is determined by the local shape of the boundary $\partial \Omega_{1}$ near $p_{1}$, up to biholomorphic equivalence. This will imply Theorem 1 , since the domains $\Omega_{1}$ and $\Omega_{2}$ near $p_{1}$ and $p_{2}$, respectively, are exactly the same up to biholomorphic mappings. Thus we may study $\hat{\Omega}$ with the convention that $\Omega=\Omega_{1}$ and $p=p_{1}=0$.

The inductive proof of the main theorems is by induction on the dimension. The complex two dimensional case will be proved first. Then we will carry out 
the proof in complex dimension three using the result in dimension two. Then we will describe the induction on complex dimension $n$ in general.

We now prove Theorems 1 and 2 in complex dimension two:

We may assume that the domain $\Omega$ is convex near $0 \in \partial \Omega$. Also, let

$$
\Omega=\left\{(z, w) \in \mathbf{C}^{2}: \rho(z, w)<0\right\}
$$

with $\rho(0,0)=0$ and let the tangent plane to $\partial \Omega$ at 0 be defined by $\operatorname{Im} z=0$. Then we may also assume

$$
\rho(z, w)=u+\sum_{i+j=2 k} c_{i j} w^{i} \bar{w}^{j}+O\left(v^{2}, v w, w^{2 k+1}\right)
$$

near 0 , where $u=\operatorname{Re} z, v=\operatorname{Im} z$, and $c_{i j} \in \mathbf{C}$.

Then clearly we can find positive real numbers $C_{1}, C_{2}$ and domains $D_{1}, D_{2}$, respectively, such that, for some neighborhood $U$ of 0 in $\mathbf{C}^{2}, D_{1} \cap U \subset \Omega \cap U \subset$ $D_{2} \cap U$ with $\{0\} \subset \partial D_{1} \cap \partial \Omega \cap \partial D_{2}$, where

$$
\begin{aligned}
& D_{1}:=\left\{(z, w) \in \mathbf{C}^{2}: \operatorname{Re} z<-C_{1}|w|^{2 k}\right\}, \\
& D_{2}:=\left\{(z, w) \in \mathbf{C}^{2}: \operatorname{Re} z<-C_{2}|w|^{2 k}\right\} .
\end{aligned}
$$

Let $\left\{A_{j}\right\}$ be the sequence of complex $n$ by $n$ matrices in Lemma A. Then, the sequence consisting of the terms $a_{l m}^{k}=\left\langle A_{k} v_{l}, v_{m}\right\rangle_{\text {Euc }}$, where $v_{l}$ is the vector in $\mathbf{C}^{n}$ whose components are all zero except the $l$ th component which is 1 , can be assumed, by extracting a subsequence if necessary, to satisfy $\left|a_{11}^{j} / a_{21}^{j}\right| \geq C>$ 0 for some constant $C>0$. Then we will have $a_{21}^{j} / \sqrt[2 k]{\left|a_{22}^{j}\right|}$, and $a_{22}^{j} / \sqrt[2 k]{\left|a_{11}^{j}\right|}$ bounded for all $j$, since, otherwise, either $D_{2}$ collapses to a set with empty interior or $D_{1}$ becomes too big to be hyperbolic at the limit, an obvious contradiction to Lemma A. Hence there is a "unique and canonical" scaling up to complex linear equivalence, depending only on the local defining function of $\partial \Omega$ at $(0,0)$.

Moreover, we will have, at the limit,

$$
\operatorname{Re}(z+h w)<-P_{2 k}(c z+d w)
$$

as a defining inequality of $\hat{\Omega}$, where $h, c$ and $d$ are complex numbers and where $P_{2 k}(w)=\sum_{i+j=2 k} c_{i j} w^{i} \bar{w}^{j}$. (Note that all the higher order terms vanish at the limit.) Therefore, $\Omega$ is biholomorphic to the domain defined by the inequality $\operatorname{Re} \zeta<-P_{2 k}(\xi)$. This proves Theorems 1 and 2 in complex dimension 2.

Now we prove Theorems 1 and 2 in complex dimension 3, using the results in complex dimension 2. By Condition (L) and Lemma A, we may assume that $\Omega$ is actually convex near $p$. Then since $\partial \Omega$ is of type $2 k$ at $p=0$, the defining function $\rho$ of $\Omega$ near $p=0$ can be written as

$$
\begin{aligned}
\rho\left(z_{1}, z_{2}, z_{3}\right)= & \operatorname{Re} z_{1}+P_{m_{2}}\left(z_{2}\right)+P_{m_{3}}\left(z_{3}\right)+Q_{n_{1} n_{2}}\left(z_{2}, z_{3}\right) \\
& +O\left(v_{1}^{2}, v_{1} z_{2}, v_{1} z_{3}, z_{2}^{m_{2}+1}, z_{3}^{m_{3}+1}, z_{2}^{n_{1}+1} z_{3}^{n_{2}}, z_{2}^{n_{1}} z_{3}^{n_{2}+1}\right)
\end{aligned}
$$


where

(a) $v_{1}=\operatorname{Im} z_{1}$

(b) $m_{2}=2 k$,

(c) $P_{m_{l}}(l=1,2)$, is a homogeneous polynomial in $z_{l}$ and $\bar{z}_{l}$ with degree $m_{l}$,

(d) $Q_{n_{1} n_{2}}$ consists of all monomials of degree $n_{1}$ in $z_{2}, \bar{z}_{2}$, and of degree $n_{2}$ in $z_{3}, \bar{z}_{3}$, respectively, and,

(e) $n_{1}>0$ and $n_{2}>0$.

This expression is easily obtained by virtue of the convexity of $\Omega$ at $p=0 \in$ $\partial \Omega$. Now we try to find $\hat{\Omega}$ explicitly by a direct computation. We introduce the following notations for the later convenience:

$A_{j} \in G L_{n}(\mathbf{C})$ the scaling sequence introduced in Theorem A.

$B_{j}:=A_{j}^{-1}=\left(b_{\alpha \beta}^{j}\right)$.

$\Omega_{j}=A_{j}(\Omega)$, assuming that $p=0 \in \mathbf{C}^{n}$.

$\hat{\Omega}=\lim _{j \rightarrow \infty} \Omega_{j}$ as before.

Then $\partial \Omega_{j}$ is defined by

$$
\begin{aligned}
0= & \operatorname{Re}\left(b_{11}^{j} z_{1}+b_{12}^{j} z_{2}+b_{13}^{j} z_{3}\right) \\
& +P_{m_{2}}\left(b_{21}^{j} z_{1}+b_{22}^{j} z_{2}+b_{23}^{j} z_{3}\right)+P_{m_{3}}\left(b_{31}^{j} z_{1}+b_{32}^{j} z_{2}+b_{33}^{j} z_{3}\right) \\
& +Q_{n_{1} n_{2}}\left(b_{21}^{j} z_{1}+b_{22}^{j} z_{2}+b_{23}^{j} z_{3}, b_{31}^{j} z_{1}+b_{32}^{j} z_{2}+b_{33}^{j} z_{3}\right)+O^{j},
\end{aligned}
$$

in coordinates $\left(z_{1}, z_{2}, z_{3}\right)$, where:

$$
\begin{aligned}
O^{j}=O\left(\left(v_{1}^{j}\right)^{2}, v_{1}^{j} z_{2}^{j},\right. & v_{1}^{j} z_{3}^{j},\left(z_{2}^{j}\right)^{m_{2}+1},\left(z_{3}^{j}\right)^{m_{3}+1}, \\
& \left.\left(z_{2}^{j}\right)^{n_{1}+1}\left(z_{3}^{j}\right)^{n_{2}},\left(z_{2}^{j}\right)^{n_{1}}\left(z_{3}^{j}\right)^{n_{2}+1}\right),
\end{aligned}
$$

with

$$
z_{h}^{j}=\sum_{k=1}^{3} b_{h k}^{j} z_{k} \quad \text { for } h=1,2,3, \text { and } v_{1}^{j}=\operatorname{Im} z_{1}^{j} .
$$

We may assume, without loss of generality, that

$$
\left|b_{1 k}^{j}\right| /\left|b_{11}^{j}\right| \leq \text { constant, for any } j=1,2,3, \ldots,
$$

for each $k=1,2,3$. Also we may assume that $b_{11}^{j}>0$ for all $j$, replacing $A_{j}$ by $\left(b_{11}^{j} /\left|b_{11}^{j}\right|\right) A_{j}$ after extracting a subsequence from $\left\{A_{j}\right\}$ if necessary so that the sequence $b_{11}^{j} /\left|b_{11}^{j}\right|$ is convergent. Then we prove:

Lemma B. The sequence $\left\{b_{l k}^{j} / \sqrt[m_{2}]{b_{11}^{j}}\right\}$ is bounded for all possible $l, k$ and $j$. Proof. Since $\Omega$ is convex at $p=0$ and is of finite type $m_{2}$, there is an open neighborhood $N$ of 0 in $\mathrm{C}^{n}$ such that $U \cap N \subset E_{C} \cap N$, where

$$
E_{C}=\left\{\left(z_{1}, z_{2}, z_{3}\right) \in \mathbf{C}^{3} \mid \operatorname{Re} z_{1}<-C\left(\left|z_{2}\right|^{m_{2}}+\left|z_{3}\right|^{m_{3}}\right)\right\} \text {. }
$$


Therefore, considering the fact that, for fixed $R>0, \Omega_{j} \cap B_{R} \subset A_{j}\left(E_{C}\right) \cap B_{R}$ for any $j$ large, it is clear that $\lim _{j} A_{j}\left(E_{C}\right)$ must contain some open set. But then $A_{j}\left(E_{C}\right)$ is given by the inequality

$$
\begin{aligned}
\operatorname{Re}\left(b_{11}^{j} z_{1}+\right. & \left.b_{12}^{j} z_{2}+b_{13}^{j} z_{3}\right) \\
& <-C\left(\left|b_{21}^{j} z_{1}+b_{22}^{j} z_{2}+b_{23}^{j} z_{3}\right|^{m_{2}}+\left|b_{31}^{j} z_{1}+b_{32}^{j} z_{2}+b_{33}^{j} z_{3}\right|^{m_{2}}\right)
\end{aligned}
$$

i.e.,

$$
\begin{aligned}
& \operatorname{Re}\left(z_{1}+\frac{b_{12}^{j}}{b_{11}^{j}} z_{2}+\frac{b_{13}^{j}}{b_{11}^{j}} z_{3}\right) \\
& <-C\left(\left|\frac{b_{21}^{j}}{\sqrt[m_{2}]{b_{11}^{j}}} z_{1}+\cdots+\frac{b_{23}^{j}}{\sqrt[m_{2}]{b_{11}^{j}}} z_{3}\right|^{m_{2}}\right. \\
& \left.+\left|\frac{b_{31}^{j}}{\sqrt[m_{2}]{b_{11}^{j}}} z_{1}+\cdots+\frac{b_{33}^{j}}{\sqrt[m_{2}]{b_{11}^{j}}} z_{3}\right|^{m_{2}}\right) .
\end{aligned}
$$

Therefore, to have $\lim _{j} A_{j}\left(E_{C}\right)$ contain some open set, we must have the righthand side bounded. So the lemma follows.

To understand the proof more geometrically and intuitively, we assume that the tangent pianes $T_{0}\left(\partial \Omega_{j}\right)$ converge in the sense of the local Hausdorff distances in $\mathbf{C}^{n}$. By the lemma above, we assume that $b_{l k}^{j} / \sqrt[m_{2}]{b_{11}^{j}}$ converges for any $l=2,3, k=1,2,3$, and that $b_{1 l}^{j} / b_{11}^{j}$ converges for $l=2,3$. Then consider

$$
\Omega^{\prime}:=\left\{\left(z_{1}, z_{2}, z_{3}\right) \in \Omega \mid z_{3}=0\right\}
$$

which is a complex two dimensional section of $\Omega$, represented by

$$
0>\operatorname{Re} z_{1}+P_{m_{2}}\left(z_{2}\right)+O\left(v_{1}^{2}, v_{1} z_{2}, v_{1} z_{3}, z_{2}^{m_{2}+1}\right),
$$

Then it is clear that $\hat{\Omega}$ contains $\lim _{j} A_{j}\left(\Omega^{\prime}\right)$ in its closure. Note that $\lim _{j} A_{j}\left(\Omega^{\prime}\right)$ will be defined by

$$
\operatorname{Re}\left(z_{1}+a z_{2}+b z_{3}\right)<-P_{m_{2}}\left(\alpha z_{1}+\beta z_{2}+\gamma z_{3}\right),
$$

as in (5), where $a, b, \alpha, \beta$ and $\gamma$ are complex numbers. Since $\hat{\Omega}$ is a convex domain in $\mathbf{C}^{3}$, if the vectors $(1, a, b)$ and $(\alpha, \beta, \gamma)$ are $\mathbf{C}$-linearly dependent, $\hat{\Omega}$ will contain a complex line. This violates the fact that $\hat{\Omega}$ has to be hyperbolic in Kobayashi's sense. Therefore, the vectors

$$
(1, a, b)=\lim _{j}\left(1, b_{12}^{j} / b_{11}^{j}, b_{13}^{j} / b_{11}^{j}\right)
$$

and

$$
(\alpha, \beta, \gamma)=\lim _{j}\left(\frac{b_{21}^{j}}{\sqrt[m_{2}]{b_{11}^{j}}}, \cdots, \frac{b_{23}^{j}}{\sqrt[m_{2}]{b_{11}^{j}}}\right)
$$

are linearly independent over $\mathbf{C}$. 
So we have the following two cases to consider, knowing that $\hat{\Omega}$ exists:

Case 1. $P_{m_{3}}\left(\sum_{k=1}^{3} b_{3 k}^{j} z_{k}\right) / b_{11}^{j}$ is bounded.

Case 2. $P_{m_{3}}\left(\sum_{k=1}^{3} b_{3 k}^{j} z_{k}\right) / b_{11}^{j}$ tends to $\infty$ as $j \rightarrow \infty$.

We will show that, in Case 1 , there is a unique $\hat{\Omega}$, up to biholomorphic equivalence, determined entirely by the local defining function of $\Omega$ at $p \in \partial \Omega$ which is the boundary point satisfying Condition (L). Also, we will show that Case 2 does not occur. This will complete the proof of Theorem 1 . Theorem 2 will be obtained by observing what the limit has to be in this procedure.

Case 1. In this case, as we pointed out before, $\left\{b_{3 k}^{j} / \sqrt[m_{3}]{b_{11}^{j}}\right\}$ has to be a bounded sequence and hence may be assumed to be convergent by extracting a subsequence for each $k=1,2,3$. Then expression (2) gives us, at the limit, the defining equation of $\partial \hat{\Omega}$ :

$$
\begin{aligned}
0= & \operatorname{Re}\left(z_{1}+a z_{2}+b z_{3}\right)+P_{m_{2}}\left(\alpha z_{1}, \beta z_{2}, \gamma z_{3}\right)+P_{m_{3}}\left(s z_{1}, t z_{2}, r z_{3}\right) \\
& +\lim _{j \rightarrow \infty}\left\{Q_{n_{1} n_{2}}\left(\sum_{k=1}^{3} b_{2 k}^{j} z_{k}, \sum_{k=1}^{3} b_{3 k}^{j} z_{k}\right) / b_{11}^{j}+O^{j} / b_{11}^{j}\right\},
\end{aligned}
$$

where $O^{j}$ and $a, b, \alpha, \beta, \gamma$ are as in (2a) and (10a,b), respectively, and where

$$
(s, t, r)=\lim _{j \rightarrow \infty}\left(\frac{b_{31}^{j}}{\sqrt[m_{3}]{b_{11}^{j}}}, \frac{b_{32}^{j}}{\sqrt[m_{3}]{b_{11}^{j}}}, \frac{b_{33}^{j}}{\sqrt[m_{3}]{b_{11}^{j}}}\right) .
$$

Since the right-hand side of (11) has to be finite as a whole, we must have $Q_{n_{1} n_{2}} / b_{11}^{j}$ bounded, because it is the term which grows fastest, if it diverges in this case. But then

$$
\begin{aligned}
Q_{n_{1} n_{2}}\left(\sum_{k=1}^{3} b_{2 k}^{j} z_{k}, \sum_{k=1}^{3} b_{3 k}^{j} z_{k}\right) / b_{11}^{j} \\
\quad=Q_{n_{1} n_{2}}\left(\sum_{k=1}^{3} \frac{b_{2 k}^{j}}{\sqrt[m_{2}]{b_{11}^{j}}} z_{k}, \sum_{k=1}^{3} \frac{b_{3 k}^{j}}{\sqrt[m_{3}]{b_{11}^{j}}} z_{k}\right) \cdot\left(b_{11}^{j}\right)^{\frac{n_{1}}{m_{2}}+\frac{n_{2}}{m_{3}}-1}
\end{aligned}
$$

and hence we must have $\frac{n_{1}}{m_{2}}+\frac{n_{2}}{m_{3}}-1 \geq 0$ and the $Q$ term will converge either to 0 or $Q_{n_{1} n_{2}}\left(\alpha z_{1}+\beta z_{2}+\gamma z_{3}, s z_{1}+t z_{2}+r z_{3}\right)$, depending on the defining function $\rho$. Then $\hat{\Omega}$ will be defined by, up to a holomorphic change of coordinates,

$$
0>\tilde{\rho}\left(\zeta_{1}, \zeta_{2}, \zeta_{3}\right)=\operatorname{Re} \zeta_{1}+P_{m_{2}}\left(\zeta_{2}\right)+P_{m_{3}}\left(\zeta_{3}\right)+\tilde{Q}\left(\zeta_{2}, \zeta_{3}\right)
$$

where $\tilde{Q}$ is either 0 or $Q_{n_{1} n_{2}}$ with $\frac{n_{1}}{m_{2}}+\frac{n_{2}}{m_{3}}=1$ depending on $\rho$. So Case 1 is now completely understood.

Remark. If the vectors $(1, a, b),(\alpha, \beta, \gamma),(s, t, r)$ are linearly dependent over $\mathbf{C}$, then $\hat{\Omega}$ would contain a complex line, which is not allowed. 
Case 2. We now prove that no scaling by $\left\{A_{j}\right\}$ is possible in this case.

Since we know that $\hat{\Omega}$ exists, the following expression for $\partial \Omega_{j}$ must have the right-hand side bounded:

$$
\begin{aligned}
0= & \operatorname{Re}\left(z_{1}+\frac{b_{12}^{j}}{b_{11}^{j}} z_{2}+\frac{b_{13}^{j}}{b_{11}^{j}} z_{3}\right) \\
& +P_{m_{2}}\left(\sum_{k=1}^{3} \frac{b_{2 k}^{j}}{\sqrt[m_{2}]{b_{11}^{j}}}\right)+P_{m_{3}}\left(\sum_{k=1}^{3} \frac{b_{3 k}^{j}}{\sqrt[m_{3}]{b_{11}^{j}}} z_{k}\right) \\
& +Q_{n_{1} n_{2}}\left(\sum_{k=1}^{3} b_{2 k}^{j} z_{k}, \sum_{k=1}^{3} b_{3 k}^{j} z_{k}\right) / b_{11}^{j}+O^{j} / b_{11}^{j},
\end{aligned}
$$

where $O^{j}$ is as in $(2 \mathrm{a})$.

Notice that we have shown that the first two terms on the right-hand side are bounded. Again by considering the rate of divergence, we can just ignore the $O$ part, because it is of lower growth rate, even though it goes to infinity. Hence, we must have

$$
\left|Q_{n_{1} n_{2}} / b_{11}^{j}\right| \nearrow \infty
$$

and

$$
\left(P_{m_{3}}+Q_{n_{1} n_{2}}\right) / b_{11}^{j} \text { is stable. }
$$

So we let

$$
T^{j}(z):=P_{m_{3}}\left(\sum_{k} b_{3 k}^{j} z_{k}\right) / b_{11}^{j}+Q_{n_{1} n_{2}}\left(\sum_{k=1}^{3} b_{2 k}^{j} z_{k}, \sum_{k=1}^{3} b_{3 k}^{j} z_{k}\right) / b_{11}^{j} .
$$

Let $z^{0}=\left(z_{1}^{0}, z_{2}^{0}, z_{3}^{0}\right) \in \hat{\Omega}$. Then there will be $\varepsilon_{0}>0$ such that $\left(1+\varepsilon_{0}\right) z^{0} \in \hat{\Omega}$ since $\hat{\Omega}$ is open. Then we have

$$
\begin{aligned}
T^{j}\left(\left(1+\varepsilon_{0}\right) z^{0}\right)=\left(1+\varepsilon_{0}\right)^{m_{3}} T^{j}\left(z^{0}\right) \\
\quad+\left[\left(1+\varepsilon_{0}\right)^{n_{1}+n_{2}}-\left(1+\varepsilon_{0}\right)^{m_{3}}\right] Q_{n_{1} n_{2}}\left(\sum_{k=1}^{3} b_{2 k}^{j} z_{k}^{0}, \sum_{k=1}^{3} b_{3 k}^{j} z_{k}^{0}\right) / b_{11}^{j}
\end{aligned}
$$

bounded. Thus we must have $m_{3}=n_{1}+n_{2}$. But then

$$
T^{j}(z)=P_{m_{3}}\left(\sum_{k=1}^{3} \frac{b_{3 k}^{j}}{\sqrt[m_{3}]{b_{11}^{j}}} z_{k}\right)+Q_{n_{1} n_{2}}\left(\sum_{k=1}^{3} \frac{b_{2 k}^{j}}{\sqrt[m_{3}]{b_{11}^{j}}} z_{k}, \sum_{k=1}^{3} \frac{b_{3 k}^{j}}{\sqrt[m_{3}]{b_{11}^{j}}} z_{k}\right)
$$

Note that $m_{3} \leq m_{2}$, since $m_{2}=\tau(\partial \Omega, 0)$. Hence the sequence

$$
\left\{\left(b_{21}^{j} / \sqrt[m_{3}]{b_{11}^{j}}, \ldots, b_{23}^{j} / \sqrt[m_{3}]{b_{11}^{j}}\right)\right\}
$$


is never bounded unless $m_{3}=m_{2}$. But in Case 2, because of Lemma B, we know that $m_{3}<m_{2}$. As we observed before

$$
\begin{aligned}
& \left(\frac{b_{21}^{j}}{\sqrt[m_{3}]{b_{11}^{j}}}, \cdots, \frac{b_{23}^{j}}{\sqrt[m_{3}]{b_{11}^{j}}}\right) \\
& \quad=\left(\frac{b_{21}^{j}}{\sqrt[m_{2}]{b_{11}^{j}}}, \cdots, \frac{b_{23}^{j}}{\sqrt[m_{2}]{b_{11}^{j}}}\right) \cdot\left(b_{11}^{j}\right)^{\frac{1}{m_{2}}-\frac{1}{m_{3}}} \rightarrow \infty \text { as } j \rightarrow \infty .
\end{aligned}
$$

So by (17) and (18), we have

$$
\begin{aligned}
T^{j}(z)= & P_{m_{3}}\left(\sum_{k} \frac{b_{3 k}^{j}}{\sqrt[m_{3}]{b_{11}^{j}}} z_{k}\right) \\
& +\left(b_{11}^{j}\right)^{n_{1}\left(\frac{1}{m_{2}}-\frac{1}{m_{3}}\right)} \cdot Q_{n_{1} n_{2}}\left(\sum_{k=1}^{3} \frac{b_{2 k}^{j}}{\sqrt[m_{2}]{b_{11}^{j}}} z_{k}, \sum_{k=1}^{3} \frac{b_{3 k}^{j}}{\sqrt[m_{3}]{b_{11}^{j}}} z_{k}\right) .
\end{aligned}
$$

Now write

$$
B_{k}^{j}=\frac{\left(b_{k 1}^{j}, b_{k 2}^{j}, b_{k 3}^{j}\right)}{\sqrt{\left|b_{k 1}^{j}\right|^{2}+\left|b_{k 2}^{j}\right|^{2}+\left|b_{k 3}^{j}\right|^{2}}} \text { for } k=2,3
$$

and assume, again choosing subsequences of $\left\{A_{j}\right\}$ if necessary, that

$$
\lim _{j \rightarrow \infty} B_{2}^{j}=B_{2} \text { and } \lim _{j \rightarrow \infty} B_{3}^{j}=B_{3} .
$$

Then if $B_{2}$ and $B_{3}$ are linearly independent over $C$, we can choose $z^{1}, z^{2} \in \hat{\Omega}$ such that $B_{3} \cdot z^{1}=B_{3} \cdot z^{2}, B_{2} \cdot z^{1} \neq B_{2} \cdot z^{2}$ and

$$
Q_{n_{1} n_{2}}\left(B_{2} \cdot z^{1}, B_{3} \cdot z^{1}\right) \neq Q_{n_{1} n_{2}}\left(B_{2} \cdot z^{2}, B_{3} \cdot z^{2}\right) \text {. }
$$

But then either $\left|T^{j}\left(z^{1}\right)\right| \nearrow \infty$, or $\left|T^{j}\left(z^{2}\right)\right| \nearrow \infty$, which is clearly a contradiction. Therefore, we have only to check the last remaining possibility that the vectors $B_{2}$ and $B_{3}$ are linearly dependent over $C$.

Let us assume that $B_{2}=\lambda B_{3}$ for some $\lambda \in \mathbf{C},|\lambda|=1$. Then (19) becomes

$$
T^{j}(z)=\Lambda_{1}^{j} \cdot P_{m_{3}}\left(B_{3}^{j} \cdot z\right)+\Lambda_{2}^{j} \cdot Q_{n_{1} n_{2}}\left(c B_{2}^{j} \cdot z, B_{3}^{j} \cdot z\right),
$$

which must converge, where $c$ is a complex number. Therefore, they must cancel out completely, because both $\Lambda_{1}^{j}$ and $\Lambda_{2}^{j}$ tend to infinity.

Now we repeat the same process on the homogeneous parts of next higher degree like $P_{m_{3}}+Q_{n_{1} n_{2}}$, and end up with the limit domain defined by the inequality

$$
0>\operatorname{Re}\left(B_{1} \cdot z\right)+P_{m_{2}}\left(B_{2} \cdot z\right)+H\left(B_{2} \cdot z, B_{3} \cdot z\right),
$$

for some real valued homogeneous polynomial $H$. But since $B_{2}=\lambda B_{3}$, this domain will possess a complex line sitting inside, which cannot be allowed by 
the hyperbolicity of $\Omega$ and Lemma A. This completes the proof in the case of complex dimension three.

Finally, we specify the induction step on $n=$ complex dimension $(\Omega)$ to finish the proof:

$\left(\mathrm{H}_{n}\right)$ With defining function

$$
\begin{aligned}
\rho\left(z_{1}, \ldots, z_{n}\right)= & 2 \operatorname{Re} z_{1}+P_{m_{2}}\left(z_{2}\right)+\cdots+P_{m_{n}}\left(z_{n}\right) \\
& +\sum_{i_{2}, \ldots, i_{n}} Q_{i_{2} \cdots i_{n}}\left(z_{2}, z_{3}, \ldots, z_{n}\right)+\text { higher order terms, }
\end{aligned}
$$

where the sum is taken over the $(n-2)$-tuples $\left(i_{2}, \ldots, i_{n}\right)$ of nonnegative integers satisfying

$$
\frac{i_{2}}{m_{2}}+\cdots+\frac{i_{n}}{m_{n}}=1
$$

and where $P_{m_{k}}$ and $Q$ are as described in Theorem 2, the scaling sequence satisfies the following conditions:

(1) $b_{1 l}^{j} / b_{11}^{j}$ and $b_{k l}^{j} / \sqrt[m_{k}]{b_{11}^{j}}$ are bounded regardless of $j$, for each $k=2,3$ and $l=1,2,3$.

(2) $b_{11}^{j}>0$.

(3) The vectors $B_{1}, \ldots, B_{n}$ defined by

$$
\begin{aligned}
& B_{1}=\lim _{j}\left(1, b_{12}^{j} / b_{11}^{j}, \ldots, b_{1 n}^{j} / b_{11}^{j}\right), \\
& B_{k}=\lim _{j}\left(b_{k 1}^{j} / \sqrt[m_{2}]{b_{11}^{j}}, \ldots, b_{k n}^{j} / \sqrt[m_{2}]{b_{11}^{j}}\right) \quad(k=2, \ldots, n)
\end{aligned}
$$

are linearly independent over $\mathbf{C}$.

Now the induction step is completed by the same argument we have used to prove $\left(\mathrm{H}_{3}\right)$ assuming $\left(\mathrm{H}_{2}\right)$. This completes the proof of all the results stated in $\S 2$.

\section{REFERENCES}

1. J. D'Angelo, Real hypersurfaces, orders of contact, applications, Ann. of Math. 115 (1982), 615-637.

2. S. Frankel, Bounded convex domains with compact quotients are symmetric spaces in complex dimension two, Thesis, Stanford Univ., 1986.

3. R. E. Greene and S. G. Krantz, Deformation of complex structures, estimates for the $\bar{\partial}$ equation, and stability of the Bergman kernel, Adv. in Math. 43 (1982), 1-86.

4. Characterizations of certain weakly pseudoconvex domains with non-compact automorphism groups, Complex Analysis, Seminar, University Park, Pa., 1986, Lecture Notes in Math., vol. 1268, Springer-Verlag, 1987.

5. S. Kobayashi, Hyperbolic manifolds and holomorphic mappings, Marcel Dekker, New York, 1970.

6. S. Pinchuk, Holomorphic inequivalences of some classes of domains in $\mathbf{C}^{n}$, Math. USSR Sb. 39 (1981), 61-86. 
7. J.-P. Rosay, Sur une caracterization de la boule parmi les domaines de $\mathbf{C}^{n}$ par son groupe d'automorphismes, Ann. Inst. Fourier (Grenoble) 29 (1979), 91-97.

8. B. Wong, Characterization of the ball in $\mathbf{C}^{n}$ by its automorphism group, Invent. Math. 41 (1977), 253-257.

Department of Mathematics, Brown University, Providence, Rhode Island 02912 